\title{
The Foundation of Traditional Chinese Medicine
}

\author{
Patrick Kim Cheng Low, Sik-Liong Ang \\ Universiti Brunei Darussalam Brunei Darussalam, Brunei \\ Email: patrick_low2003@yahoo.com,angsikliong@gmail.com \\ Received August 31, 2010; revised September 17, 2010; accepted September 30, 2010
}

\begin{abstract}
In this paper, the authors examine and interpret the concept of Traditional Chinese Medicine (TCM) whom the Chinese believes and practices for so many centuries. The authors also explain the ancient Chinese concepts of Chi and Tao (Yin and Yang) which are the foundation of the TCM. This paper also seeks to discuss the ways of attaining a healthy body with the clarity of mind as well as to demonstrate the benefits of such healthy lifestyle.
\end{abstract}

Keywords: Traditional Chinese Medicine (TCM), Chinese Concepts of Tao (Yin and Yang) and Chi, Meditation, The Yellow Emperor's Canons of Internal Medicine, The Tao of Revitalization

\section{Introduction}

Medicine-the science of healing the sick has been practiced for thousands of years. Even the earliest human had used plants and herbs as medicines and had tried simple surgical procedures, such as putting splint on broken bones. Over the last two hundred years, we have made huge progress in the clinical and surgical procedures in medicine [1]. Chinese medicine which is also considered as an alternative medicine is gradually being accepted and is practiced even in the Western world.

\subsection{The Paper's Aim \& Objectives}

In this paper, the aim and objectives are to illustrate the concept of Traditional Chinese Medicine (TCM) which is of paramount importance for one to achieve a healthy lifestyle. The authors interpret and explain that the ancient Chinese concepts of Chi and Tao (Yin and Yang) are the foundation of the TCM. The paper also seeks to discuss the ways of attaining a healthy body with the clarity of mind as well as to demonstrate the benefits of such healthy lifestyle.

\subsection{What is Chinese Medicine?}

Chinese Medicine is also called Traditional Chinese Medicine (TCM). It includes a range of traditional medicine practices originating in China. TCM practices include treatments such as Chinese herbal medicine, acupuncture, dietary therapy, and also both the Tui $\mathrm{Na}$ and
Shiatsu massages. Chi Kung (Qigong) and Tai Chi movements (Taijiquan) are also closely associated with TCM. TCM has been practiced by the Chinese for thousands of years and is rooted in meticulous observation of how nature, the cosmos, and the human body are interacting. Major theories include; Tao (Yin and Yang), Chi, the Five Phases (Wu Xing), the human body Meridian/ hannel system and Zang Fu organ theory.

\subsection{What is Tao? What is Yin and Yang}

Lao Tzu, was one of the earliest philosopher in the Chinese history, who describes the marvel of Tao as an evolving force that operates throughout the universe. Tao is the first cause of the universe. Lao Tzu said that Tao is 'the way' and he emphasized this in the first verse of his Tao Teh Ching [2] that:

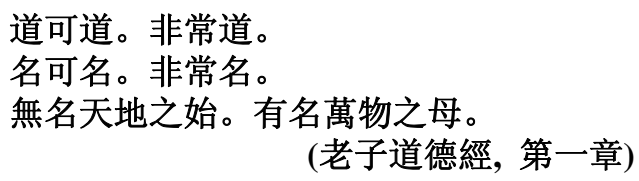

Translated as:

The Tao that can be said is not the everlasting Tao. If a name can be named, it is not the everlasting name. That which has no name is the origin of heaven and earth; That which has a name is the mother of all things.

(Lao Tzu’s Tao Teh Ching, Verse.1) [3] 
Therefore Tao is always without a name and that it is the origin of heaven and earth. Tao can also be said to be the "Absolute" that it can be said to be the movement and a stillness without a beginning, Yin and Yang (also known as Tai Chi) are things that can be said to be without a beginning (Cleary 2003).

The Tai Chi (Ultimate Principle of Existence) involved "The two dynamic powers" (the white space represents the Yang and the black space represents the Yin) exists in equilibrium and from which a coordinated and vigorous force is produced. [4]

This classic symbol for Yin and Yang appears like a pair of fish swimming in a circle around each other; the tail of one is formed from the head of the other. Here, we can see that Yin-Yang are born out of each other and are transformed into each other. Each of the Yin-Yang contains the seed of the other; there is a tiny seed circle of dark Yin contained in the white part of Yang, as there is a seed circle of white Yang contained in the darkness of Yin. Tao is the force, which flows through all lives. Each person is to nurture the breathing or what is also known as the integral life force ("Chi” or "Qi") that has been given to him/her. Unlike Western thinking, time is not linear but cyclical. And overall, each and every Taoism believer's goal is to align him(her)self, by having a balance (the perfect sense of balance is embodied in the idea of Yin-Yang) or being harmonious with the Tao. In the universe, there should always be a balance of nature. Ying (female) and Yang (male) are always at work, and there should be a good balance between them; and hence the avoidance of extremes. This is indeed what the concept of Traditional Chinese Medicine (TCM), which is anchored in Taoism roots, is based on. The fact that one who knows how to maintain good health, one would always carry out one's daily life in accordance to nature. Thus, one would need to follow the principle of Yin and Yang and keeps in conformity with the art of predicting the consequences of what would happen based on the interaction of Yin and Yang. By doing so, one would beable to modulate or transform one's life in harmony with nature. By way of recuperating the essence and the vital energy, one would then master and practice the way of

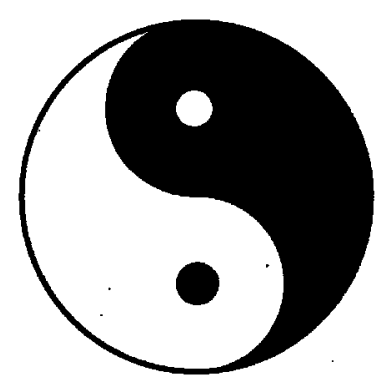

Figure 1. Tai Chi diagram. maintaining harmony as well as good health. For instance, if one's behavior in daily life is kept in regular patterns including one's food and drink intake, its fixed amount, as well as one's daily activities where one would not overwork one's body. And if, one is also based on Taoism practices, it is taken that one should be aware and, in fact, be sensitive of the balance of Yin and Yang in nature. One can also take it as axiomatic that in the morning after a good night sleep, one is often refreshed and energized. In the morning when the Yang (Chi or energy) is at its high and at its abundance, one becomes naturally active, thereby, as one uses one's energy, one gets tired. One needs to balance the Yang with Yin (taking rest or naps) during the day so that one maintains one's energy level (This is very true when one gets older). When the evening comes, the Yin (Chi) becomes abundant or overwhelming; one then has to conserve the Yang energy as well in balance to be ready for rest. If the Yang energy is used in a way that it is in an extreme manner, one's whole body is not in balance and one becomes totally exhausted so much so that one may not even rest well. One, thus, needs to follow the way of nature and be in balance. Also, in the same way, one cannot pull the seedling to assist its growth. Thus, the Chinese saying: "to pull seedlings to help them grow", meaning to work hard in a self-defeating way - or going against nature for quick results; and this is foolish. The Western equivalent here is that of "penny wise and pound foolish”. And in fact, in everything, nature must take its course. And by understanding the balance of Yin and Yang in nature with regards to the daily living, one could live a healthier life in body and in spirit.

\subsection{Chi and the Balance of Yin and Yang}

Western Medicine is different from the TCM because the TCM has a concept of Chi as a form of energy. It is believed that this energy exists in all things (living and non-living) including air, water, food and sunlight. Chi is said to be the unseen vital force that nourishes one's body and sustains one's life. It is also believed that an individual is born with an original amount of Chi at the beginning of one's life and as one grows and lives, one acquires Chi from eating and drinking, from breathing the surrounding air and also from living in one's environment. An individual would become ill or dies if one's Chi in the body is imbalanced or exhausted.

When one studies the principle of the Life Force; the Chi and the Tao (Yin and Yang); one would understand how this Life Force manifests in nature. Through self-cultivation, one basically enriches one's Chi for optimum health and longevity. This happens when one subscribes to this Life Force from nature that flows freely into one's mind and body. However, this requires one to 
live freely from desires, worries and emotions. To live freely, one has to detach from the worldly possessions. For the instance, money is to be spent, there is to-ing and fro-ing, thus a going and a coming of it; and there is a non-attachment to the money or the material things for better flow. Furthermore, one is required to discipline oneself by having a proper diet, sleep and exercise so that one would not disturb and interrupt with the movement of Life Force which may cause the Chi to dissipate in one's body. This dissipation of Chi would result one to fall into sickness, disease, physical and mental sufferings. It is the Taoist's belief that the practice of Chi Kung, Tai Chi movements and meditation helps one to harmonize one's Life force with one's environment and nature.

Stationary and Moving: One would like to ask this question: Why, for clarity of the mind, do both the Chi and the balance of stationary (Yin) and moving (Yang) are so important?

First of all, take note of it: When one is asleep, one's body is heavy and resting (stationary) and not moving. In fact, the body takes time to recreate, rejuvenate its strength or regenerate energies for the body, the storehouse of energy (Chi).

Doctors normally advise one to have enough sleep (usually 6-8 hours) and younger children require more sleep than adult (some more than 12 hours) for growth and tissue repairs. When one is short of sleep, one's mind is not clear. One may feel groggy; in fact, one normally feels tired and one cannot think straight or properly since the lack of sleep affects one's focus and concentration. If one sleeps too much, (one feels "heavy") one also feels drowsy and cannot think effectively as well.

When one is awake, one's body feels lighter, and one can move from one place and another, by spending on its kinetic energy. On one hand, too many activities will make one's body and mind feel tired and on the other hand, too few activities will also make the body and the mind feel bored.

So it is meant that there should be an optimum point when sleep (potential energy) and being awakened (kinetic energy) are balanced for the body and the mind to be effective and active.

As everything is centered on the nature, Taoism encourages man to take the path of nature because the path which nature itself would follow is not for human interference or interventions. Water flows downwards and that it is the natural flow or spontaneously natural. Forcing one's way against nature, going against the grain, forcing nature to bend to one's will is not good as it harms oneself. But by relaxing and allow nature to go its way, everything will fall into place. It is wu wei er wu bu wei-by doing nothing, everything is done.

\section{Chi Kung (Qigong) for Good Health}

The practice of Chi Kung (Qigong) is to regulate and control the Chi within the body. Chi Kung practice involves the manipulation and balance of the Chi within the practitioner's body. According to Taoism, the regulation of Chi is carried out through the three interconnected components: the Mind, the Body and the Spirit. For the Taoist, the training of the mind and the body is through meditation, contemplation and physical exercises. It sometimes also includes the ingestion of Chinese herbs to regulate one's Chi within the body. The development of Traditional Chinese Medicine has added more detailed to the Chi within the human body. In this system, Chi travels through the body along twelve main meridian channels and numerous smaller branches and tributaries. These main meridians also correspond to twelve main organs: the lung, large intestines, stomach, spleen, heart, small intestine, urinary bladder, kidney, liver, gallbladder, pericardium, and the "triple warmer,' which represents the entire torso region. The amount and flow of Chi is affected by one's emotional state which is ultimately related to the Mind, the Body and the Spirit. To put it simply, most Chi Kung practitioners use this concept of the proper Chi flow through those meridians as a basic premise.

\subsection{Tai Chi for Good Health}

Maintaining the balance of the Yin-Yang equilibrium is also the concept of Tai Chi and can be illustrated in practice by the Tao practitioners in the Tai Chi movements. Today, Tai Chi becomes a form of relaxation or destressing exercise which is sometimes known as the “Moving Meditation”. This is developed by a Taoist monk during the thirteenth century in ancient China. The principle of Tai Chi movement is based on the philosophy of Lao Tzu (Tao Teh Ching) and the Chinese medicine (TCM). In practicing Tai Chi, one performs certain prescribed movements and one must constantly maintain an upright and naturally balance posture (central equilibrium) during the process. Hence, movements which are too extreme resulting in the out of balance body posture are avoided in this exercise. By doing so, it is said that Tai Chi would help to de-stress one's body. Practicing Tai Chi regularly is said to be beneficial because the relaxation exercise also promotes steady breathing, regulates blood circulation, and relieves tension by doing gentle movement of the body. Meditation in motion is also believed to help in refreshing and replenishing one's energy to its normal level.

\subsection{Meditation for Good Health}

The tradition of resting in silence of an undistracted or clear mind is thousands of years old. It is practiced by 
seekers in all of the world's spiritual traditions, as well as in hospitals and even corporate boardrooms. Meditation has been used in the East, but only became popular in the West in the $1960 \mathrm{~s}$, after the British pop group, the Beatles, visited India. Scientists now agree that meditation could raise the levels of important chemicals in our bodies that help to fight off infections [5]. A very simple meditation technique that one can adopt is to find a stable and comfortable posture so that one can become aware of one's body in the present moment. To do so, one can sit on a chair with one's feet flat on the floor; or sit cross legged on a meditation cushion. What matters is that one has a sense of stability, comfort and ease. The next step is to bring one's awareness into the present moment and one becomes aware of one's environment and the sounds around oneself. Take a few deep breaths and relax and later on, one can then simply become aware of how the breath breathes itself in its own rhythms. This simple meditation exercise will help one to be fully aware of the present moment and minimize one's mind from wandering off to other thoughts or things. The art of the meditation is to see the wandering of the mind and to acknowledge it in the moment, and then to return to one's breathing. In some ways, meditation is a remembering or self-remembering and it is a process of waking up, of being with the breath or the body, and then to return to one's usual way when one is not in the meditating mode. As we know that thinking, planning, remembering and worrying often take up a lot of times in our living, and it is very important that we should understand the condition of our body so that we can perform in an optimum way; it is with constant practice of meditation and live more fully with lesser thinking and worrying that we would be able to have a healthy body and a healthy mind. [6]

In ancient China, scholars were very good in following the nature, waking up when the rooster crowed. They sit calmly meditating when the sun was rising; it was felt that this gave them energy for the day. Meditative awareness really reduces tension and heals (re-charges) one's body; Meditation quiets or calm one's minds and gently opens one's heart; it steadies one's spirit. [6] After reading and writing for a long time, the scholars walked into the open air admiring nature. When it is time for them to sleep, they got rid of any worries by telling themselves not to ponder on them; they cleared their mind of any concerns. Such ways, attitude or consciousness enabled them to live or experienced less stress, if not, no stress at all.

\subsection{TCM Based on Yellow Emperor's Canons of Internal Medicines}

The Yellow Emperor said, "Since ancient times, it is considered that the existence of men has depended on the interactions of Yin and Yang energies (Chi) in various proportions and in many ways, and that human life is based on Yin-Yang principles. As all things on earth and in the universe communicate through the Yin-Yang principles, therefore, human being can be considered as a small universe because the human body has everything that the universe has; all follows the Yin and Yang principles...” [7].

Extending this concept further, the Emperor remarked, "Besides these Yin and Yang energies are the foundation of life, the survival of men also depends on the five elements on earth (metal, wood, water, fire and earth), it is the so called, "Life depend on five". The five elements further correspond to the three Yins (cold, dryness and wetness) and the three Yangs (wind, fire and summer heat). It is so called, "Energies depend on the three". If one cannot balance these elements and the Yin-Yang energies, and one violates the principles of preserving health frequently, one's health will be hurt by the imbalance of the Yin and Yang energies or the negative factors and would contract disease..." For instance, based on the concept that the human energy is connected with that of the universe and that during the times when there is no strong wind or heavy rain storm, the human body will be fresh and cool in a calm environment. If one can keep one's spirit quiet by refraining from the emotions of over joy or excessive anger, one would have a peaceful mind as clear as a blue sky. By this time, one's Yang energy is substantial to guard the body against any negative factors and one would attain a healthy body. Similarly, if one has the ability of adapting oneself to the sequence and variations of any environment such as the four seasons, one would be able to preserve one's health in a good way. Conversely, when one is in extreme anger, the Yang energy and the blood would rush upward to the head. If the blood stagnates in the chest due to constant anger and emotional upset; the blood circulation and the vital energy would be obstructed. This would result in an unhealthy body and often one would contract sickness such as high blood pressure or a heart attack.

As one can clearly see, Yin and Yang are always in constant dynamic motion maintained by a continuous adjustment of the relative levels of Yin and Yang. When either Yin or Yang are out of balance, they naturally affect each other and change their proportions to achieve a new balance. And there are four possible ways in which a Yin-Yang imbalance can occur as illustrated in the diagram below:

\section{1) Preponderance or Dominance of Yin}

When Yin is excessive, it induces the decrease of Yang and it also means that the Yin consumes Yang.

\section{2) Preponderance of Yang}

When Yang is excessive, it induces the decrease of Yin and it also means that Yang consumes Yin. 


\section{3) Weaknesses of Yin}

When Yin is weak, Yang will be seen in apparent excess. This apparent excess is only in relation to the deficient quality of Yin.

\section{4) Weaknesses of Yang}

When Yang is weak, Yin will be seen in apparent excess. Similarly, this apparent excess is only in relation to the deficient quality of Yang.

Therefore, in these illustrations, it is very critical to be able to see the differences between the two states: the preponderance of Yin and the weakness of Yang. This is because on one state it is the truly excess Yin and on the other state it is the weakness of Yang that Yin is seen as apparent excess. Similarly, this differentiation applies between the preponderance of Yang and the weakness of Yin. [8]

It can be said that the theory of Yin and Yang is fundamental in Traditional Chinese Medicine (TCM) and every physiological process and every symptom or sign of a human body can be analyzed in the light of the Yin-Yang theory. In other words, TCM sees illness as an imbalance in the patient's whole system. It tries to get to the underlying root cause of a health problem. The aim is to heal the person's mind, body and spirit rather than just his or her sore throat or stomach ache. [11] Ultimately,

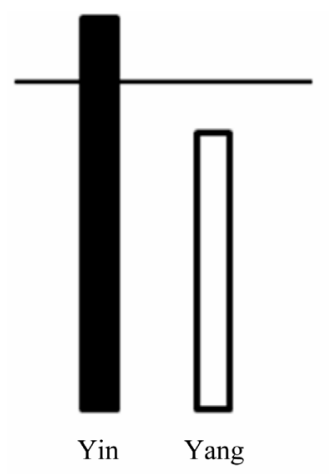

1. Preponderance of Yin

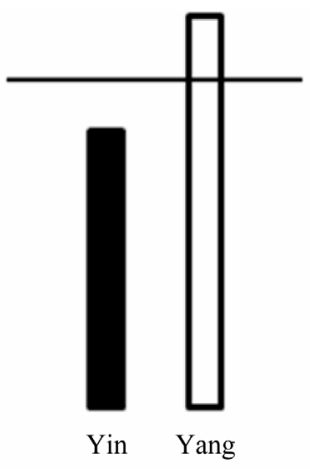

2. Preponderance of Yang
Figure 2. Preponderance of Yin and Yang.

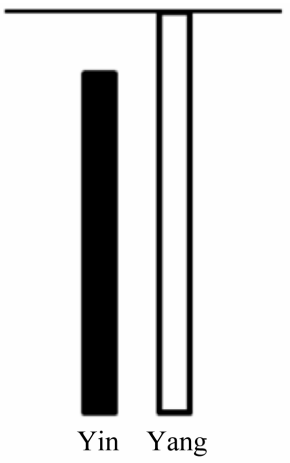

3. Weaknesses of Yin

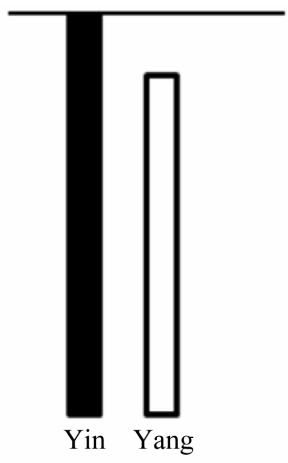

4. Weaknesses of Yang
Figure 3. Weaknesses of Yin and Yang. every treatment modality is aimed at 1) improving Yang, 2) improving Yin, 3) reducing excess Yang and reducing excess Yin, and understanding the application of the theory of Yin-Yang theory is of great importance.

\subsection{The Tao of Revitalization-The Chinese Sys- tem of Self-Healing}

To maintain, nourish, revitalize and prolong our lives, it is said that we should spend our lives simply to fulfill two basic needs namely:

1) Ingestion (eating and drinking)

2) Motion

a) Mind "Movement" (Thinking)

b) Body "Movement" (Breathing and internal organs and external limbs movement.

These two basic conditions if not fully or properly satisfied will affect one's life. For instance, if a person is not given sufficient nutrients through eating and drinking, his/her body will not be healthy and his/her life will shorten. Similarly, if one does not exercise mentally or physically, one's body will weaken. It was with these considerations that the ancient Taoists created the Tao of Revitalization which is the method of thinking, breathing, and exercising for one to maintain good health. [9] It is believed that a human body is a perfectly well-balanced, self-regenerative and self-protecting organism and that the body itself can respond to natural healing treatments instead of drug intakes. The Tao of revitalization is a series of mental and physical movements, almost effortless internal exercises that can energize the body's own life-force to repel fatigue, illness and disease and prevent them from reoccurring. Amongst all the internal exercises recommended for good health, a very simple series of physical and mental exercises that one can adopt are the five animal exercises which are related to the five elements.

Nowadays, we are living in a world full of stress for the reason that everything we do has to be fast in an urgent manner to meet daily targets and in most ways, we are living and working in a competitive, rush, rush world. Every day, most of us have little time to exercise. To spend less yet quality time to relieve or reduce stress in an efficient way, the authors here recommend these simple exercises based on the Traditional Chinese Medicine (TCM) which had been practiced by the ancient Taoists a long time ago; thus, although they are old, they indeed bear modern relevance.

\subsubsection{The Five Animal Exercises}

Taoists designed the five exercises after five animals whose movements were proven effective for the healing of human beings and they were the dragon (fire), bear (earth), eagle (metal), monkey (water) and tiger (wood). By imi- 
tating their characteristic movements human beings can alleviate the imbalanced functioning of their organs.

\subsubsection{The Dragon Exercise (Fire Element)}

The purpose of the Dragon Exercise is to instill the characteristics of the dragon into the mind and body of the practitioner. This exercise affects the mind by helping to overcome feelings of depression, anger, hostility, and all the anxieties brought on by being overwhelmed by adverse circumstances, for the dragon, flying through the heavens, is above all mundane concern.

One begins the exercise by standing still. Then, one takes a few deep breaths while imagining as vividly as possible that one is a dragon with glowing eyes, open mouth with fangs, glistening emerald scales, curling tail, paws splayed showing long claws. Then, raising one foot, assume the pose and character of a dragon. While imagining that one's hands are claws, hold one arm up with claws down and hold the other arm down with claws up. As this is not a formalized pose, a certain degree of freedom of expression is allowed within the confines of the image. Hold the pose as long as one holds the image without straining. One repeats as many times as one comfortably can. The most important aspect of this and all the other exercises is the union of the body and the mind. If the image fades or the mind wanders during the pose, one stops and begins again. No benefit will be obtained unless the body and the mind are in union.

Since the dragon represents the fire element, the physical effect of its exercise is to bring equilibrium to the heart, blood vessels, and absorption in the small intestines.

\subsubsection{The Bear Exercise (Earth Element)}

The power and strength of the bear becomes evident when it stands and walks on its hind legs. In this position, the most prominent physical feature of the bear also becomes obvious-its stomach, which protrudes outward and prevents the bear from walking straight.

One begins this exercise by standing still. One takes a few deep breaths while visualizing oneself as a bear. Then with legs stiff, stomach pushed out, arms sloping out in front, walk slowly forward. As one does this, one will feel the movement of one's abdomen and the stimulation of the area of the spleen-pancreas. One continues walking this way as long as the image remains fixed in one's mind. One repeats as many times as is convenient. The bear is associated with the earth element, and so this exercise affects the enzyme production of the spleenpancreas and the functioning of the stomach muscle. This exercise is therefore recommended for bad digestion, hyper-and hypoglycemia, and diabetes.

\subsubsection{The Eagle Exercise (Metal Element)}

To the ancient Taoists the flying eagle represented the spirit because of its god-like qualities - silence, serenity, and invisibility. The eagle is also an accomplished hunter. It soars effortlessly to great heights, and its sharp eyes are alert to all details of the landscape below. The eagle manifests its attributes of intelligence, alertness, and ease when it hunts.

One begins the Eagle Exercise by standing still. One takes a few deep breaths while imagining oneself as an eagle. When the visualization is complete, one begins to walk slowly with one's arms held out to the side in a slant, or with one's hands gently clasped behind oneself. As one walk, imagine one is an eagle, effortlessly floating through the blue sky, untouchable, divine. One's body should be very relaxed, but one's mind and eyes should be very alert, noticing everything without focusing on anyone thing in particular. One continues the exercise as long as the mind does not wander. If it does, one stops and begins again. Though this exercise can be performed anytime, anywhere, it is especially effective if done outdoors, after the evening meal.

The eagle is associated with the metal element, so the Eagle Exercise stimulates the lungs, skin, and the large intestine.

\subsubsection{The Monkey Exercise (Water Element)}

To the ancient Taoists the monkey epitomized boundless activity, curiosity, and free will. The monkey is constantly active, whether on the ground, swinging in the trees, or leaping playfully about, uninhibited by any cultural conventions.

One begins by standing or sitting. One takes a few deep

Table 1. Shows the relationship between the exercises and the organs.

\begin{tabular}{|c|c|c|c|c|c|}
\hline Elements & Fire & Earth & Metal & Water & Wood \\
\hline Exercise & Dragon & Bear & Eagle & Monkey & Tiger \\
\hline Organs & $\begin{array}{l}\text { Heart Small Intestine } \\
\text { Triple Heater ( Endo- } \\
\text { crine) Heart Constrictor } \\
\text { (Blood Vessels) }\end{array}$ & $\begin{array}{l}\text { Spleen-pancreas } \\
\text { Stomach Muscle }\end{array}$ & $\begin{array}{l}\text { Lungs Large Intes- } \\
\text { tine Skin }\end{array}$ & $\begin{array}{l}\text { Kidneys } \\
\text { Bladder } \\
\text { Bones }\end{array}$ & $\begin{array}{l}\text { Liver Gallbladder } \\
\text { Nerves }\end{array}$ \\
\hline
\end{tabular}


breaths while imagining oneself as a monkey. When the visualization is complete, one kicks off one's shoes, throws off one's clothes, and begins to act like a little monkey. One sits on the floor, crouches in a chair, leaps about, bounces up and down, hangs upside down or by one arm, whatever is physically possible to do without strain or exertion. This exercise is completely free- style; all the movements and actions should act out impulses and whims as they occur to one. Monkeys also rub and scratch themselves a great deal. One may do this also, especially in the area of the kidneys. As the embodiment of free will, the monkey inspires an exercise that is free-style in the broadest sense. This exercise is best done in private as the presence of others might be inhibiting.

The monkey is associated with the water element, so the Monkey Exercise stimulates the functions of the kidneys and bladder. This exercise is recommended for those feeling confined or restricted by circumstances in which there is a lack of freedom. To the Taoist will power resides in the kidneys. The Monkey Exercise is also recommended for any problems involving the kidneys, bladder, and urinary tract.

\subsubsection{The Tiger Exercise (Wood Element)}

The tiger demonstrates its power in its ability to capture something by leaping over it and mauling it. The tiger pose is an imitation of this "leaping over" movement.

One begins by standing still. One takes a few deep breaths while imagining oneself as a tiger. When the visualization is complete, bend one's knees slightly and rise up on one's toes while reaching up and out until one's arms are straight. Keep the claws down, as if one has reached over and out to grab something. One maintains this position as long as one can hold the image without straining the body. One repeats as many times as is comfortable.

Since tiger represents the wood element, this exercise is recommended for healing and detoxifying the liver, to sooth inflamed nerves, to balance gallbladder functions, and to detoxify the brain and body cells.

\section{Conclusion}

The ancient concepts of Chi and Tao (Yin and Yang) are the foundation of Traditional Chinese Medicine (TCM) and accordingly, disease or sickness is caused by a disruptive flow of energy or the imbalance of the Yin and Yang energies around our human bodies. Hence, TCM provides a holistic treatment meaning the whole person is being treated - his or her body, mind and spirit. It is believed that mind-body systems such as Meditation, Chi Kung (Qigong) and Tai Chi exercises could send the mind into an altered state to harness its healing power. The Tao of revitalization aims to energize, train and strengthen the internal organs so that they may become strong and healthy. The exercises also make the circulatory system run smoothly without pressuring the heart in speeding up the heart rate and that all the exercises are carried out slowly according to one's ability.

\section{References}

[1] A. Rooney, "Medicine, Stem Cells, Genes and Superbeams,” Harcourt Education, UK, 2006.

[2] M.-J. Cheng, "Lao-Tzu, My Words Are Very Easy to Understand: Lectures on the Tao Teh Ching," North Atlantic Books, Richmond, California, 1981.

[3] Lao-tzu, “Tao Te Ching,” translated by S. Mitchell, 2010 http://acc6.its.brooklyn.cuny.edu/ phalsall/texts/taote-v3. html

[4] P. K. C. Low and S.-L. Ang "Taoism and Corporate Social Responsibility,” S. O. Idowu, Ed., Encyclopaedia of Corporate Social Responsibility, Springer.(in press)

[5] C. Wallerstein, "Need to Know Alternative Medicine," Harcourt Education, UK, 2003.

[6] J. Kornfield, “Meditation for Beginners,” Bantam Books, UK, 2004.

[7] L. S. Wu and Q. Wu, "Yellow Emperor's Canon of Internal Medicine, Original Note Wang Bing (Tang Dynasty)," China Science \& Technology Press, Beijing, 1997.

[8] G. Maciocia, "The Foundation of Chinese Medicine: A Comprehensive Text for Acupuncturists and Herbalist," Churchill Livingston, London, 1989.

[9] S. Chang, "The Chinese System of Self- Healing,” Tao Publishing, USA, 1989. 